\title{
Numerical and experimental analysis of vertical spray control patternators
}

\author{
F. Sarghini, ${ }^{1}$ G. Pergher ${ }^{2}$ \\ ${ }^{1}$ University of Naples Federico II, Dept. Of Agriculture, Portici (NA), Italy; ${ }^{2}$ University of Udine, \\ DISA, Udine, Italy
}

\begin{abstract}
The experimental vertical spray control walls have the purpose of picking up the liquid delivered by trained sprayer for providing the liquid distribution profile in height. Theoretically this should correspond to the ideal profile, which consists in a uniform distribution on the vegetation. If the profile is different from the ideal, a parameter setup is required on the sprayer. Nonetheless, some problems are hidden in the aforementioned statements: i) no wall measures exactly the distribution profile (i.e. the flow through the sections in the vertical plane, parallel to the direction of advancement of the sprayer). Compared to real profile, sensitive errors are introduced: the evaporation of the drops, the deviation of the air flows caused by the sensors panel themselves; by the possibility that the drops bounce on the wall panels, also due to the current of air that can push the liquid veil laterally or upwards, Moreover, everything varies depending on the geometry of the sensors, air velocity, air humidity; ii) no one knows what exactly is the optimal distribution profile. It is often considered as optimal a profile that reflects the amount of leaf area subtended by each section absorber: however, it is evident that the path of the droplets changes according to the sprayer typology (eg. radial-flow or horizontal flows). In this work a combined numerical-experimental approach is adopted, in order to assess some of the aforementioned issues: numerical data obtained by using computational fluid dynamics models are compared and validated with experimental data, in order to assess the reliability of numerical simulations in configurations which are difficult to analyze using an experimental setup.
\end{abstract}

\section{Introduction}

The distribution of pesticides through traditional trailed sprayers, the most common configuration for this this type of agricultural oper-

Correspondence: F. Sarghini, DIAAT, Via Università, 100, 80055 Portici (NA), Italy.

E-mail: sarghini@unina.it

Key words: deposition, patternator, numerical simulation, sprayer, Vineyard.

CC Copyright F. Sarghini and G. Pergher, 2013

Licensee PAGEPress, Italy

Journal of Agricultural Engineering 2013; XLIV(s2):e73

doi:10.4081/jae.2013.s2.e73

This article is distributed under the terms of the Creative Commons Attribution Noncommercial License (by-nc 3.0) which permits any noncommercial use, distribution, and reproduction in any medium, provided the original author(s) and source are credited. ation, represents an unresolved problem because of complicated interactions between operating parameters like the air flow rate and the pesticide nozzle orientation and constructive related parameters such as the fan geometry or the nozzle topology used for the atomization. In addition, pesticide distribution in the open field further complicates the framework, through the effects due to the advancement speed or the presence of wind.

This often involves a considerable deposition off-target, with consequences not only on treatment efficiency itself but also involving a serious environmental impact and safety for the operator.

Sprayer calibration is generally considered as an essential factor in determining the effectiveness of spray application in agriculture. Proper calibration should provide a constant deposit of the pesticide per unit of target area. In spray applications to orchards or vineyards, the spatial distribution of leaves fruits or other parts of the plant may vary considerably depending on the crop, growing system, growth stage, purpose of the application, or other factors (Pergher, 2004). Therefore, calibration should include a proper adjustment of the directions of spray and air flows in order to provide a good deposition uniform.

One possibility consists in calibrating the sprayers in a control station, where pesticide distribution is analyzed using experimental vertical spray control walls or patternators; based on the liquid distribution of the pesticide some adjustment can be done, working on nozzle orientation for example. Nonetheless such approaches rely on a certain degree of heuristic intuition, due to the complexity of the flow field dynamics.

Because of the complexity of the experimental approach, in these cases the numerical modeling is an investigative tool almost indispensable. In this work an experimental validation of the numerical approach is presented and some details of the flow field are analyzed.

\section{Materials and methods}

Numerical liquid droplet tracking in trained atomizer flow field is a very complex task. As a matter of fact, the dispersion of small inertial particles in inhomogeneous turbulent flow has been long recognized as crucial in a number of industrial applications and environmental phenomena: mixing, combustion, spray dynamics, pollutant dispersion, or cloud dynamics are all example of such phenomena involving droplet transport.

In all these problems accurate predictions are important, but they not trivial to obtain because of the complex phenomenology controlling turbulent particle dynamics.

Direct numerical simulations (DNS) of turbulence coupled with Lagrangian particle tracking (LPT) (Wang and Maxey,1993; Uijttewaal and Oliemans, 1996; Rouson and Eaton, 2001) have demonstrated their capability to capture the physics of particle dynamics in relation with turbulence dynamics and have highlighted the key role played by inertial clustering and preferential concentration in determining the rates of particle interaction, settling, deposition, and entrainment. 
Due to the computational requirements of DNS, however, analysis of applied problems characterized by complex geometries and high Reynolds numbers demands alternative approaches.

Turbulent flows are characterized by eddies with a wide range of length and time scales. The largest eddies are typically comparable in size to the characteristic length of the mean flow (for example, shear layer thickness). The smallest scales are responsible for the dissipation of turbulence kinetic energy.

It is possible, in theory, to resolve directly resolve the whole spectrum of turbulent scales using an approach known as direct numerical simulation (DNS), where no turbulence modeling is required . However, DNS is not feasible for practical engineering problems involving high Reynolds number flows. The cost required for DNS to resolve the entire range of scales is proportional to $\mathrm{Re}^{3}$, where Re is the turbulent Reynolds number, but for high Reynolds numbers the cost becomes prohibitive.

In Large Eddy Simulation (LES) large eddies are resolved directly, while small eddies are modeled. The rationale behind LES can be summarized saying that momentum, mass, energy, and other passive scalars are transported mostly by large eddies, although some problems arise when particles or droplet interacts with the filtered flow field (Bianco et al, 2012). Large eddies are related to the geometries and boundary conditions of the modeled flow, while small eddies tend to be more isotropic, and are consequently more universal. As a consequence the possibility of finding an universal turbulence model is much higher for small eddies. In this work a scale similar model was adopted inside the framework of LES approach, (see Sarghini et al.,1999, for a summary of LES equations and scale similar models, and Sarghini et al, 2003 for the current implementation)

\section{Droplet modeling}

After the spray is generated from the nozzle, secondary droplet breakup (Fritsching, 2004; Markus and Fritsching, 2006) can appear. This break-up is mainly caused by the aerodynamical forces acting on the droplets and is classified by the Weber number. A droplet exposed to a relative velocity difference will eventually break up if the Weber number is large enough. The time it takes to deform and disrupt a droplet is described by the characteristic break-up time which is a result of Rayleigh-Taylor or Kelvin-Helmholtz (Levich, 1962; Bradley, 1973 ) instabilities. The spheroidization time for a droplet (Nichiporenko and Naida, 1968; Markus and Fritsching, 2006) after break-up is comparably small and hence the new droplets can be assumed to be spherical immediately after break-up. Depending on the initial Weber number, the droplets will deform and eventually break up in different sized fragments. Obviously, a high Weber number leads to a very disruptive break-up, while the break-up for small Weber numbers is slower and less disruptive. The different break-up regimes are to be distinguished by different sub-models.

In the numerical simulation several particle interaction models were introduced for particle secondary breakup, collision and coalescence.

\section{Secondary breakup: Taylor analogy model}

The Taylor analogy breakup (TAB) model (Taylor, 1981) is a classic method for calculating droplet breakup, which is applicable to many engineering sprays. This method is based upon Taylor's analogy between an oscillating and distorting droplet and a spring mass system.

The external force is given by aerodynamic droplet drag force, the damping force is due to the viscous forces, and the restoring force of the spring is given by surface tension forces

\section{Droplet collision and coalescence: O’Rourke algorithm}

Collision calculation is a time consuming task, considering that for $\mathrm{N}$ droplets, each one has $\mathrm{N}-1$ possible collision partners, setting the number of possible collision pairs to $0.5 \mathrm{~N}^{2}$ approximately. The algorithm of O'Rourke (O'Rourke, 1993), a stochastic estimate of collisions, reduces the computational cost of the spray calculation assuming that two parcels may collide only if they are located in the same continuous-phase cell. This method can be reasonably adopted only when the continuous-phase cell size is small compared to the size of the spray, being in this case the method second-order accurate at estimating the chance of collisions.

\section{Droplet size}

A Rosin-Rammler distribution was adopted, with an average $\mathrm{D}_{50}$ size equal to $250 \mathrm{~mm}$ and limit diameters $D_{10}=100 \mathrm{~mm}$ and $D_{90}=500 \mathrm{~mm}$ (Nuyttens et al, 2009) .

The numerical solver adopted in this work is OpenFoam (OpenFoam v2.2.0), a free, open source CFD software package. Several mesh configurations were tested, ranging from $300 \times 10^{3}$ up to $3 \times 10^{6}$ control volumes, and grid independence was tested on simplified test cases.

\section{Results}

Numerical results were compared with experimental data obtained from ENAMA certification report 05-109b - Poly 800/8 for an Agricolmeccanica SrL Poly 1000/8 trained atomizer. In particular, data related to liquid distribution were compared with numerical results obtained by collecting data on 2 virtual vertical spray control wall (P1 and P2, see Figure 1), positioned at $1.25 \mathrm{~m}$ from the symmetry plane on the sprayer and considering all the particle passing through a $4.15 \mathrm{~m}$ high $\times 2 \mathrm{~m}$ window during $20 \mathrm{~s}$ of computational time interval after the transient startup of the fan.

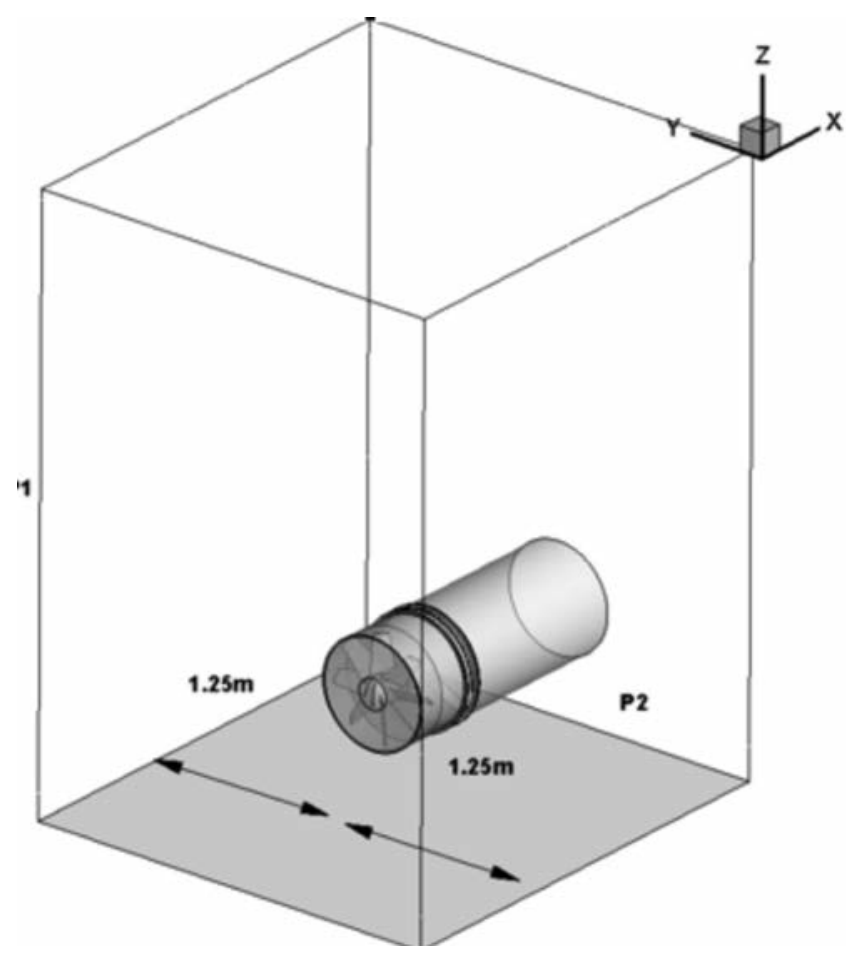

Figure 1. Numerical virtual vertical captation wall P1 and P2. 
Such data were normalized using a captation index, computed at the end of the numerical measurement, showing the collected volume (vi, in $\mathrm{ml}$ ) as a function of the measuring range for each virtual collecting panel as a percentage of the maximum value detected.

$$
d_{i}=100 \frac{v_{i}}{v_{i(\max )}}
$$

where $\mathrm{v}_{\mathrm{i}}$, in $\mathrm{ml}$, is the volume captured by each captation panel $\mathrm{i}$ and $\mathrm{V}_{\mathrm{i}(\max )}$, in $\mathrm{ml}$, is the volume collected by the captation panel providing the maximum volume.

Operative parameters considered in the simulation are: axial fan rotational speed 1836 giri/min, nozzle mass flow rate /water) 1.06 liter/min at 10 bar, total volumetric fan flow rate $33800 \mathrm{~m} 3 / \mathrm{h}$.

In Figure 2 a comparison between experimental and numerical cap-

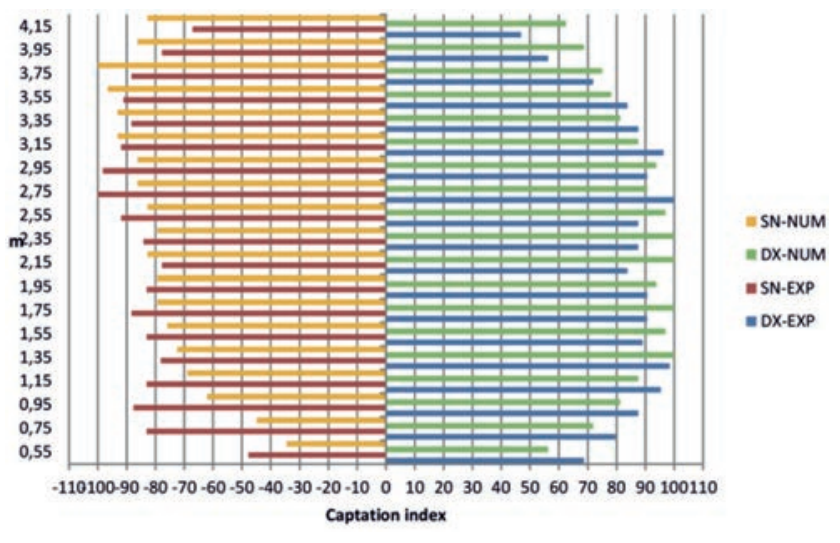

Figure 2. Experimental and numerical captation index diagram.

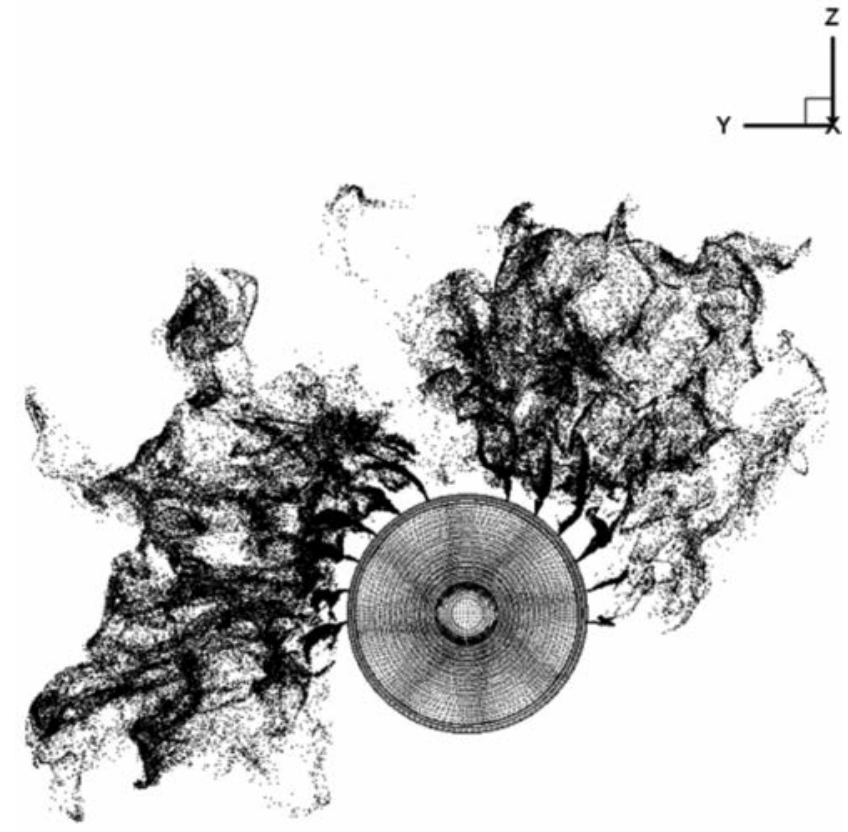

Figure 3. Droplets distribution (frontal view) - axial fan without swirl control (rotor only). itation index is presented, showing a good agreement with an asymmetric distribution between the two sides, although a small quantitative divergence is present. Moreover, numerical results tends to distribute the droplets in the higher panels of the captation wall, probably due to the presence of a secondary break-up phenomena.

In Figures 3 and 4 frontal and lateral views of droplets distribution for a tracking time equal to $1 \mathrm{~s}$ are presented. Notice that the geometrical axial fan configuration, without any device to straighten the internal flux and to reduce the swirl due to blades rotational movement, generates a strong asymmetry which is responsible of the difference in results between right and left side showed in Figure 2. Moreover, axial momentum generates also the dispersion of the droplets also on an inclined radial cone, as showed in Figure 4.

In Figure 5 and 6 velocity contours are shown on both frontal and lateral planes, showing that although at a certain distance from the nozzle

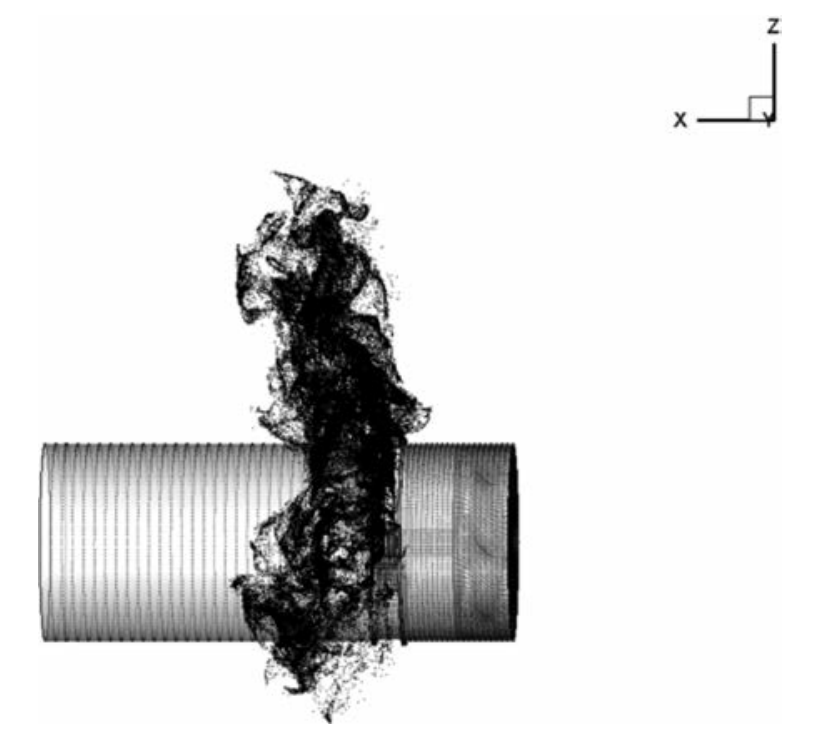

Figure 4. Droplets distribution (lateral view) - axial fan without swirl control (rotor only).

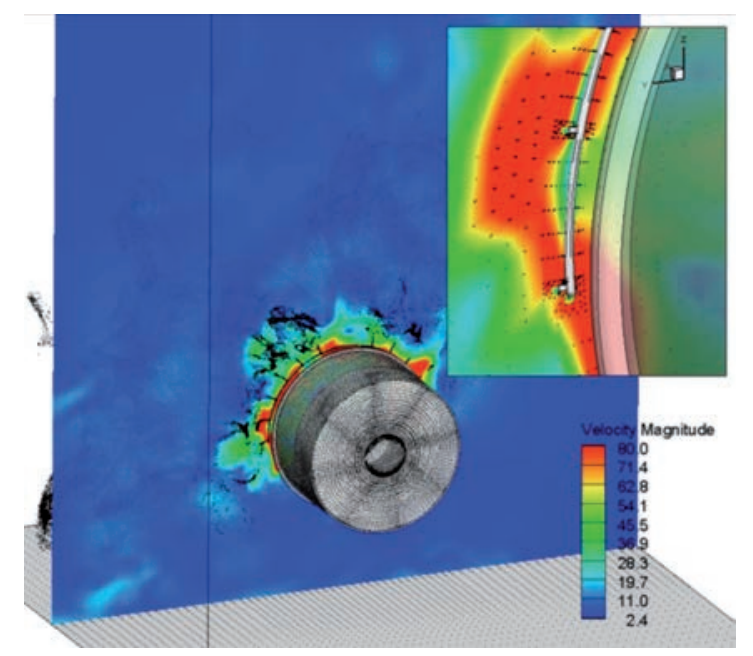

Figure 5. Velocity contour distribution (frontal view) - axial fan without swirl control (rotor only). 


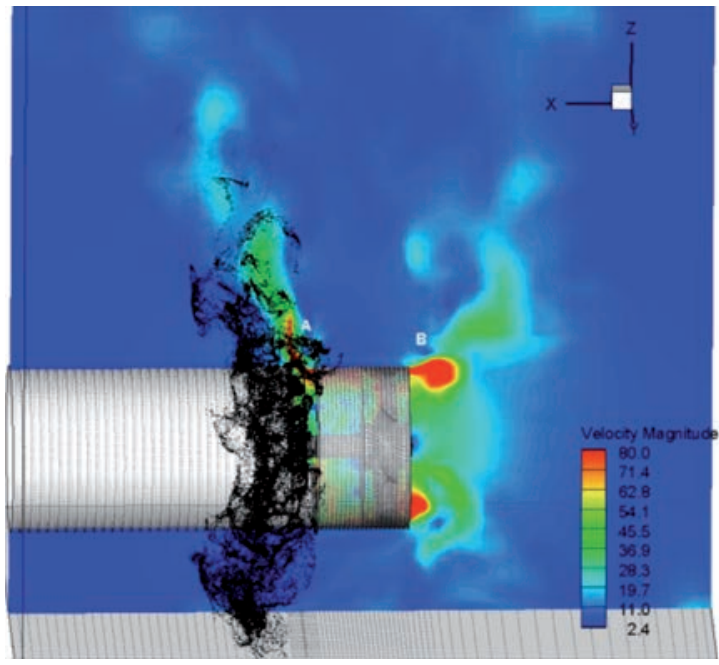

Figure 6. Velocity contour distribution (lateral view) - axial fan without swirl control (rotor only).

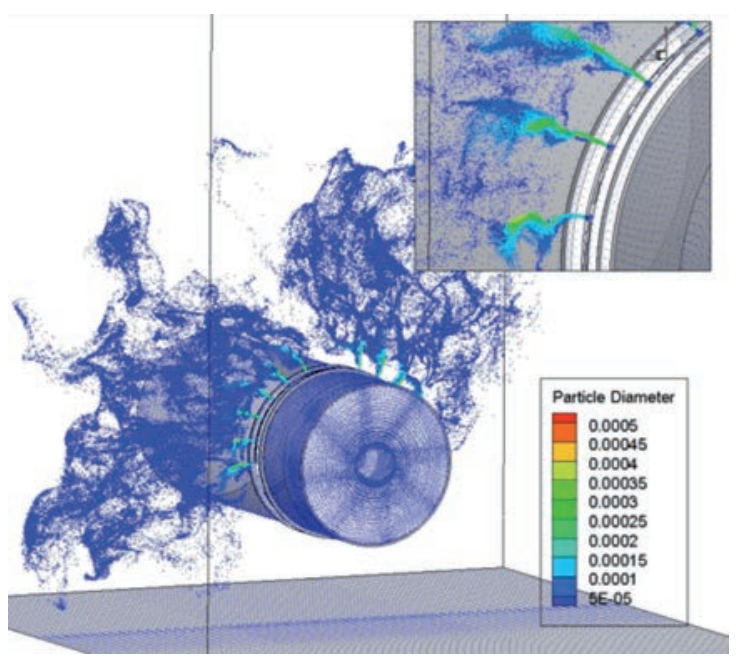

Figure 7. Droplets size distribution (frontal view) - axial fan without swirl control (rotor only).

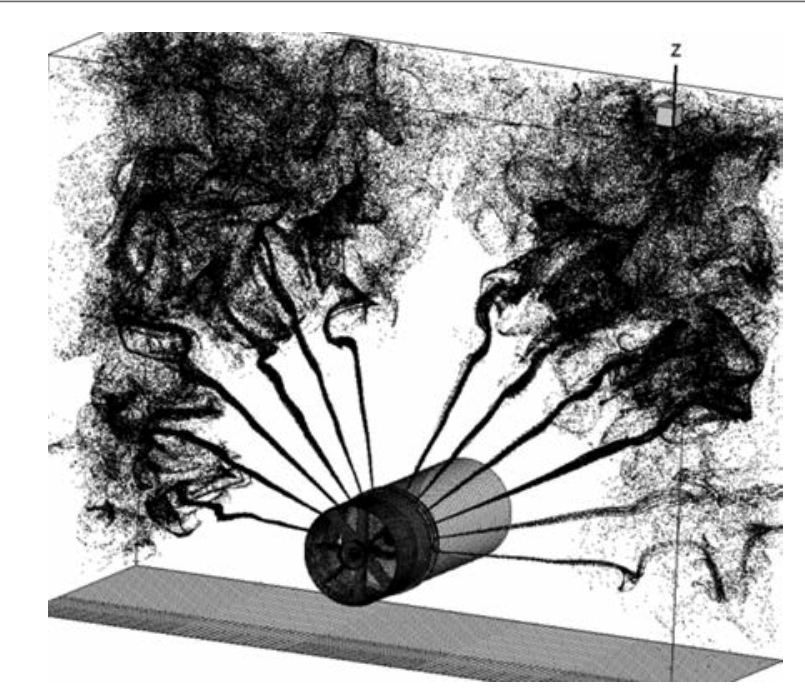

Figure 8. Droplets distribution - axial fan without moving part (uniform inflow) and direction control (stator only). ring, air speed is order of $10 \mathrm{~m} / \mathrm{s}$, near the nozzle air speed peaks are higher than $80 \mathrm{~m} / \mathrm{s}$, generating 2 main effects: an unsteady vortex shedding (Figure 6) and a secondary droplets break-up, generating a different droplets diameter distribution as showed in Figure 7.

In Figure 8 droplets distribution is showed for a simulation on a slight different configuration: rotating blades are replaced by a uniform inflow velocity distribution followed by a static blades geometry (stator), where swirl effects can be neglected: in this case vortex shedding and related particles entrainment is strongly reduced, and radial asymmetry disappears.

\section{Conclusions}

Results obtained using computational fluid dynamics technique showed a good agreement with experimental data, although a slight difference is still present. This difference can be attributed to geometrical details and secondary break-up modeling. In any case, ad-hoc experiments should be carried out in order to match all geometrical and operative parameter details.

Nonetheless, numerical experiments showed that a main role is played by the internal flow field of the axial fan, which is responsible for the unsteady flow and related vortex shedding in proximity of the nozzle's ring, inducing a backward radial bending of the nozzle jet. Another detail to be assessed is the influence of the fan inflow flow field with the flow field near the nozzle in case of short fan configuration, which is related to axial fan blade geometry.

The presence of unsteady vortices generates a strong vortex entrainment where a high number of droplets collision coalescence happens because of vortex entrainment.

Results showed that numerical simulation can be used to design more efficient patternators, allowing to gain an important insight in droplet - flow field interactions, almost impossible to obtain in an experimental setup.

\section{References}

Bianco F., Chibbaro S., Marchioli C., Salvetti M. V., and Soldati A., 2012. Intrinsic filtering errors of Lagrangian particle tracking in LES flow fields, Phys. Fluids, 24, 1.370- 1378.

Bradley D., 1973. On the atomization of liquids by high-velocity gases, Part 1. J. Phys. D: Appl. Phys. 6:1724-1736, and Part 2., J. Phys. D: Appl. Phys. 6:2267-2272.

Fritsching U., 2004. Spray Simulation: Modelling and Numerical Simulation of Sprayforming Metals. Cambridge University Press, Cambridge, UK.

Fritsching U., Zhang H., Bauckhage K.,1994. Numerical simulation of temperature distribution and solidification behaviour during spray forming, Steel Res., 65,273-278.

Markus S., Fritsching U., Bauckhage K., 2002. Jet break up of liquid metals in twin fluid atomization, Materials Science and Eng., A 326:122-133.

Nichiporenko S, Naida YI.1968. Heat exchange between metal particles and gas in the atomization process. Soviet Powder Metall. Metal Ceramics, 7(7):509-512.

Nuyttens, D., Schampheleire, M. de, Verboven, P., Brusselman, E. Dekeyser, D., 2009. Droplet size and velocity characteristics of agricultural sprays ,Transactions of the ASABE, Vol. 52 No. 5 pp. 1471-1480.

0'Rourke P. J. ,1981. Collective Drop Effects on Vaporizing Liquid Sprays". PhD thesis. Princeton University, Princeton, New Jersey. 
OpenFoam, The free CFD Toolbox, www.openfoam.org. , accessed 20.04.2013.

Pergher G., 2004, Field evaluation of a calibration method for air-assisted sprayers involving the use of a vertical patternator, Crop Protection, 23, 5 , Pages 437-446.

Rouson D. W. and Eaton J. K., 2001. On the preferential concentration of solid particles in turbulent channel flow, J. Fluid Mech. 428, 149.

Sarghini, F., U. Piomelli,, Balaras E.,1999. Scale-Similar Models for Large-Eddy Simulations, Physics of Fluids, 11, 6:1596.

Sarghini, F., De Felice, G., Santini, S., 2003, Neural networks based subgrid scale modeling in large-eddy simulations, Computers and Fluids, 32,1:97-108.
Taylor G. I. , 1963. The Shape and Acceleration of a Drop in a High Speed Air Stream, Technical Report. In the Scientific Papers of G. I. Taylor. ed., G. K. Batchelor.

Levich VG, 1962. Physicochemical Hydrodynamics. Prentice Hall, Upper Saddle River, NJ, 501-667.

Uijttewaal W. S. J. and Oliemans R. W. A.,1996. Particle dispersion and deposition in direct numerical and large eddy simulations of vertical pipe flows, Phys. Fluids 8, 2590.

Wang L. P. and Maxey M. R., 1993. Settling velocity and concentration distribution of heavy particles in homogeneous isotropic turbulence, J. Fluid Mech., 256, 27. 